\title{
Charles Bonnet syndrome and Terson's syndrome from subarachnoid hemorrhage: good news from bad news
}

\author{
Colleen M. Cebulla, \\ Havener Eye Institute, Department of Ophthalmology and Visual Sciences, The Ohio State \\ University, 915 Olentangy River Rd, Suite 5000, Columbus, OH 43212, USA
}

Christopher Minning,

Department of Internal Medicine, Riverside Methodist Hospital, 3535 Olentangy River Rd, Columbus, $\mathrm{OH} 43214$, USA

Cedric Pratt, and Havener Eye Institute, Department of Ophthalmology and Visual Sciences, The Ohio State University, 915 Olentangy River Rd, Suite 5000, Columbus, OH 43212, USA

\author{
Martin Lubow \\ Havener Eye Institute, Department of Ophthalmology and Visual Sciences, The Ohio State \\ University, 915 Olentangy River Rd, Suite 5000, Columbus, OH 43212, USA
}

Colleen M. Cebulla: colleen.cebulla@osumc.edu

\section{Dear Editor}

Charles Bonnet syndrome (CBS) has drawn recent attention because of its connection to macular degeneration and its response to improved vision with treatment [1-3].

Here we describe visual hallucinations in a 57-year-old patient with visual loss after bilateral vitreous hemorrhages due to Terson's syndrome from rupture of a cerebral aneurysm. Recognition and treatment of her vitreous hemorrhage altered her rehab management, response to care, and neurologic prognosis from that of long-term disability to normal cognition and return to her technical career.

The patient was a 57-year-old healthy woman employed as a computer technology specialist, who was found unresponsive at work from rupture of a cerebral aneurysm shown by emergency hospital studies. Images indicated subarachnoid, cisternal, and ventricular blood with hydrocephalus, attributable to a right posterior inferior cerebellar artery (PICA) aneurysm (Fig. 1). She tolerated emergency ventriculostomy, and then aneurysm repair by clipping on the following day. She was discharged to a skilled nursing facility on post-op day 17 after tracheostomy and feeding tube placement. There she was noted to have visual problems with concerns of cortical blindness, and transferred to a rehab facility on post-op day 31. Rehabilitation progressed poorly, which was attributed to cortical damage. Again, visual problems and impaired cognition were noted. She complained of confusion, and of seeing familiar objects and people that she knew were not really there. Her visual acuity was limited to light perception in each eye, found to be due to dense vitreous hemorrhage in both

(C) Springer-Verlag 2012

Correspondence to: Colleen M. Cebulla, colleen. cebulla@osumc. edu.

Conflict of Interest None.

Disclosures None. 
eyes. Pars plana vitrectomy was performed in the right eye 42 days after onset, with rapid improvement in her apparent cognition and in her response to rehab therapy the next day. Visual acuity in the right eye was 20/40 and hallucinations were gone. Vitrectomy for the left eye was done 1 month later. Final visual acuity was 20/30 right eye and 20/20 left eye (Fig. 2), with return to full-time work 3 months after the acute aneurysm rupture and bilateral vitreous hemorrhage of Terson's syndrome.

To our knowledge, this is the first case of CBS in a patient with vitreous blood from Terson's syndrome. This patient's story of significant but transient CBS is of special interest, in that the vitreous hemorrhages of Terson's syndrome and associated severe visual loss were initially unrecognized in the setting of acute coma, emergency neurosurgical procedures, and concern for neurological deficits with loss of cognitive function after cerebral hemorrhages with hydrocephalus.

Thereafter, her poor response to rehabilitation, visual hallucinations, and unrecognized vitreous hemorrhage misled observers to concerns of cortical blindness, and of cognitive loss with permanent disability. The patient's age was not that of the elderly patients in whom CBS hallucinations are more often described. Happily, despite the delayed return of vision, and the commonly limited prognosis for hallucinations in Charles Bonnet syndrome, this patient improved rapidly. Vitrectomy dramatically restored good vision, aided cognition, improved response to rehab therapy and sped her return to normal life.

\section{Acknowledgments}

The Ohio State University, Department of Ophthalmology and Visual Sciences, Havener Eye Institute research fund and KL2RR025754 from the National Center for Research Resources (CMC).

\section{References}

1. Abbott EJ, Connor GB, Artes PH, Abadi RV. Visual loss and visual hallucinations in patients with age-related macular degeneration (Charles Bonnet syndrome). Invest Ophthalmol Vis Sci. 2007; 48:1416-1423. [PubMed: 17325191]

2. Singh A, Sorensen TL. The prevalence and clinical characteristics of Charles Bonnet syndrome in Danish patients with neovascular age-related macular degeneration. Acta Ophthalmol. 2010 Epub ahead of print. 10.1111/j.1755-3768.2010.02051.x

3. Singh A, Sorensen TL. Charles Bonnet syndrome improves when treatment is effective in agerelated macular degeneration. British J Ophthalmol. 2011; 95:291-292. 

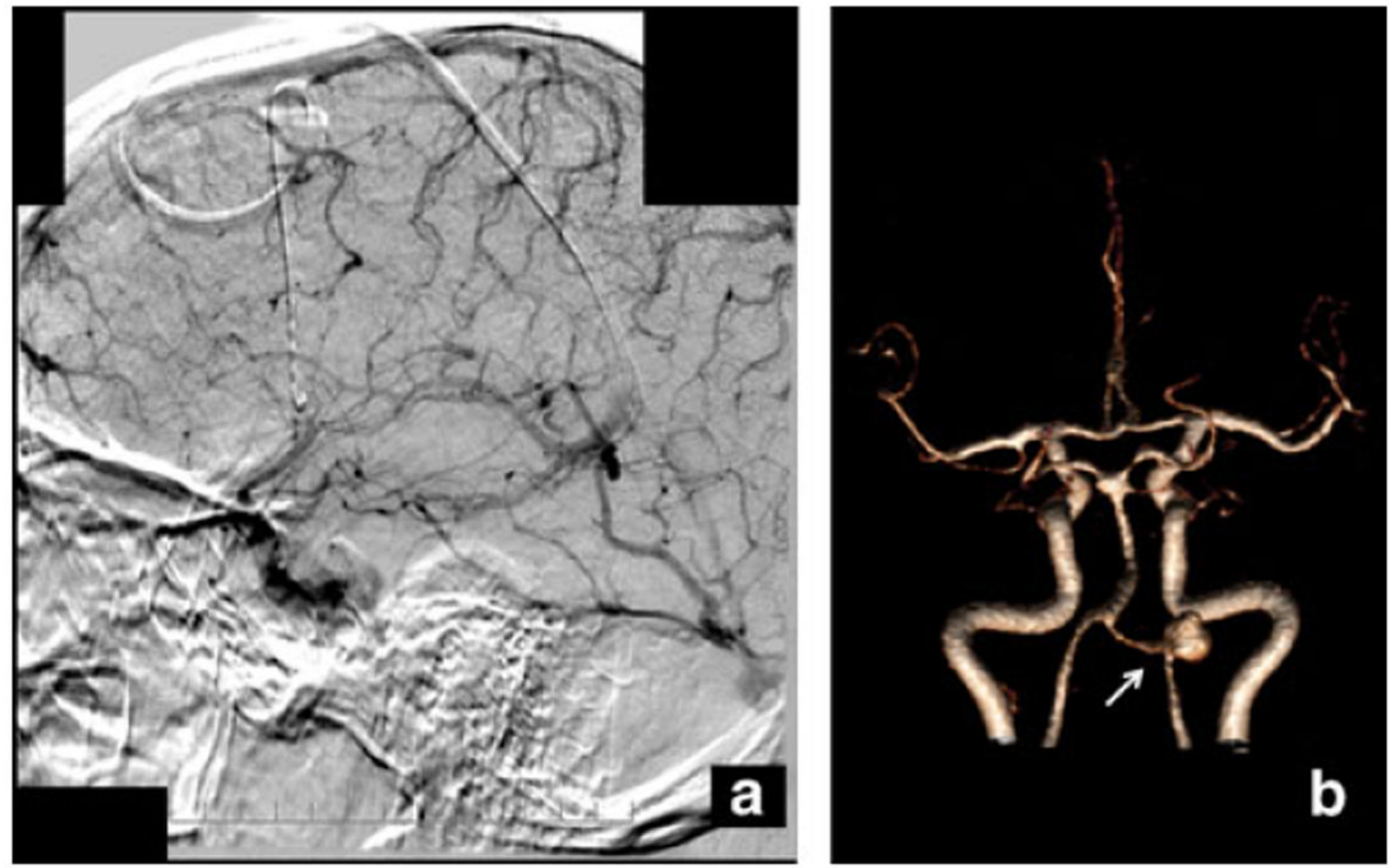

Fig. 1.

a Angiography demonstrating aneurysm. b MRA 3-D reconstruction rotated to demonstrate right PICA aneurysm (arrow) 

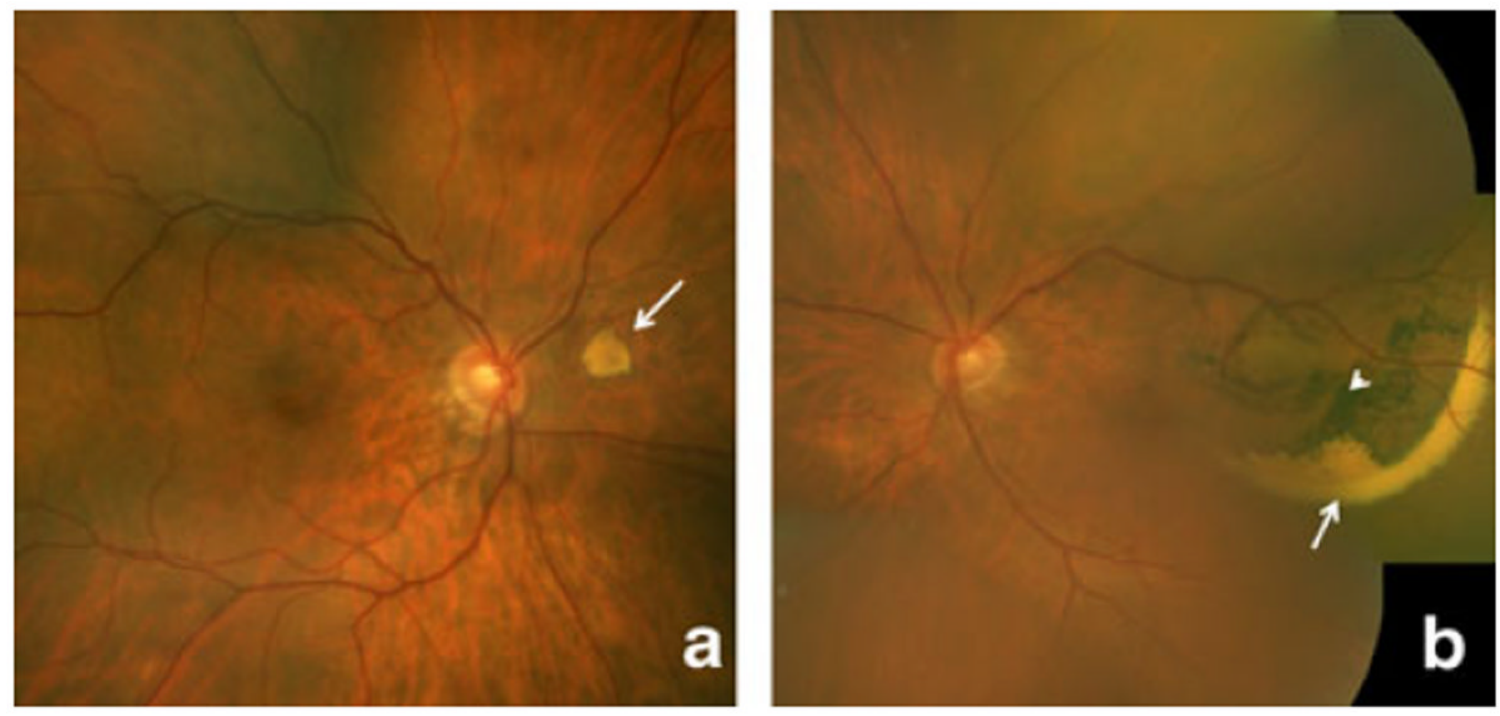

Fig. 2.

a, b Ocular fundus photographs after vitrectomy demonstrating residual dehemoglobinized subretinal hemorrhage (arrows) and pigment changes (arrowhead) 